Article

\title{
Experimental Investigations on Wavefront Distortion of LD-Pumped Neodymium-Doped Silica-Glass Rod with High Thermal Shock Parameter
}

\author{
Long Pan ${ }^{1,2}{ }^{\oplus}$, Wenfa Huang ${ }^{1, *}$, Shikai Wang ${ }^{3}$, Shengzhe Ji ${ }^{1,2}$, Xinghua Lu ${ }^{1}$, Jiangfeng Wang ${ }^{1}$, \\ Wei Fan ${ }^{1}$ and Xuechun $\mathrm{Li}^{1}$ \\ 1 Joint Laboratory on High Power Laser and Physics, Shanghai Institute of Optics and Fine Mechanics, \\ Chinese Academy of Sciences, Shanghai 201800, China; panlong1@siom.ac.cn (L.P.); szji@siom.ac.cn (S.J.); \\ luxingh@foxmail.com (X.L.); wajfeng@163.com (J.W.); fanweil@siom.ac.cn (W.F.); \\ lixuechun@siom.ac.cn (X.L.) \\ 2 Center of Materials Science and Optoelectronic Engineering, University of Chinese Academy of Sciences, \\ Beijing 100049, China \\ 3 The R\&D Center for High Power Laser Components, Shanghai Institute of Optics and Fine Mechanics, \\ Chinese Academy of Sciences, Shanghai 201800, China; woshiwsk@163.com \\ * Correspondence: huangwf@siom.ac.cn; Tel.: +86-216-991-8164
}

Received: 29 June 2020; Accepted: 20 July 2020; Published: 22 July 2020

\begin{abstract}
The characterization of a laser diode (LD)-pumped neodymium-doped silica glass (NDSG) laser is here described. The gain performance and wavefront distortion were measured, and the thermal toughness and uniformity distribution of the material were experimentally observed. At a pumping frequency of $1 \mathrm{~Hz}$ and energy $7.79 \mathrm{~J}$, a small-signal gain of 1.16 was measured, and the wavefront distortion reached $2.67 \lambda$ (wavelength $\lambda=1053 \mathrm{~nm}$ ). At a pumping frequency of $25 \mathrm{~Hz}$ with $194 \mathrm{~W}$ power, the NDSG was still not cracked, which is consistent with its high thermal shock parameter. However, the material uniformity was relatively poor. These results indicate good prospects for the application of NDSG lasers at high energy and high repetition frequency, but the gain performance, uniformity, and other aspects affected by the manufacturing process need to be improved.
\end{abstract}

Keywords: Nd-doped silica glass; small-signal gain; wavefront distortion; thermal shock parameter; LD pump

\section{Introduction}

High-energy and high repetition frequency lasers are urgently required for applications in Optical Parametric Chirped-Pulse Amplification (OPCPA) pump sources, particle acceleration, medical treatment and material processing. The gain medium used in the laser is an important subject for consideration. Good gain media need to have high gain characteristics and maintain high optical quality. However, in order to reach high energy and high repetition frequency, the unavoidable accumulation of heat occurs in the gain medium. Only a part of the energy is used in laser amplification, and most of the energy is absorbed by the medium, generating excessive heat. This will not only cause the phenomenon of thermal lensing [1,2] with the beam quality affected, but can also cause the gain medium to rupture and be damaged.

The applications such as laser inertial confinement nuclear fusion, laser shock strengthening technology and ultrastrong ultrashort pulse laser creation require high energy and a high repetition frequency. Amongst existing laser materials, phosphate neodymium-doped glass (Nd:glass) can achieve high energy storage and output, but it cannot support high repetition frequencies. The high 
thermal conductivity of Nd-doped yttrium aluminum garnet (Nd:YAG) supports high repetition frequency operation, but it is not conducive to high energy output [3].

In recent years, the Nd-doped silica glass (NDSG) has gradually gained attention. It not only has the theoretical parameters for generating high energies [4,5], but also presents the prospect of high repetition frequency lasers because of its high thermal shock resistance parameter. As high a level as $40 \mathrm{~J}$ has been reported for the laser output energy generated by a NDSG oscillator system [6]. The preparation method of NDSG has also gradually developed, from the traditional glass melting method [7] through the chemical vapor deposition (CVD) method [8], the sol-gel method [9], the zeolite method [10], and many others. Moreover, the preparation technology is still continuously improving.

In these previous studies, the laser amplification in NDSG was based on the flash lamp pumping. In comparison, the laser diode (LD) pumping is characterized by a narrower illumination wavelength, which can more effectively match the absorption spectrum of the gain medium. The LD pumping also produces less thermal deposition, and its thermal management is better. Despite these advantages, the LD pumping has not been widely used due to cost and other factors.

This study is aimed at the characterization of the laser amplification in NDSG with LD pumping, and the description of the wavefront distortion under different clamping conditions. Then, the results of testing the high thermal toughness of NDSG and the uniformity of the material are described. Finally, the potential and current deficiencies of NDSG are discussed.

\section{Spectroscopy and Optical Properties}

The measurements to characterize the laser glass were performed in the R\&D Center for High Power Laser Components affiliated with the Shanghai Institute of Optics and Fine Mechanics, Chinese Academy of Sciences. The absorption spectrum of NDSG with a doping concentration of $0.3 \mathrm{~mol} \%$ is shown in Figure 1a. There is a peak at $802 \mathrm{~nm}$, matching the wavelength of the LD pump, which aids pump absorption. The fluorescence spectrum of NDSG with a doping concentration of $0.3 \mathrm{~mol} \%$ is displayed in Figure $1 \mathrm{~b}$. The peak wavelength is in the range of 1059-1062 nm, while the linewidth is $51.7 \mathrm{~nm}$, and the fluorescence lifetime is $376 \mu \mathrm{s}$. A large linewidth leads to advantages in ultrafast laser applications.

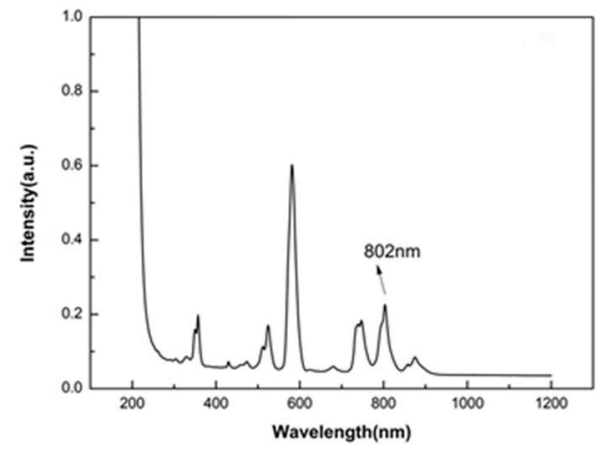

(a)

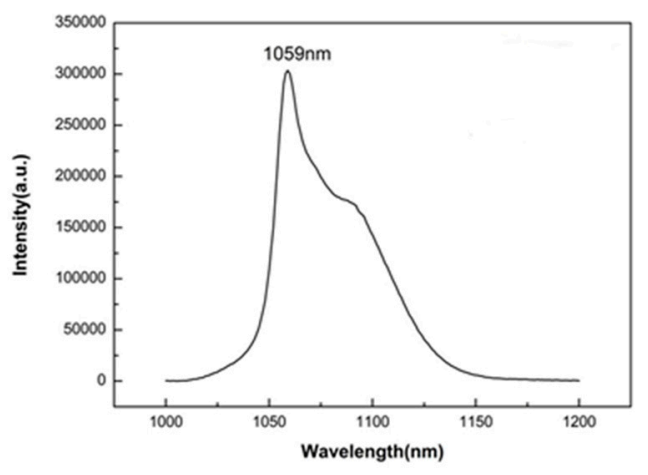

(b)

Figure 1. (a) Absorption spectrum and (b) fluorescence spectrum of NDSG with a doping concentration of $0.3 \mathrm{~mol} \%$.

For comparison, some of the physical parameters of three gain materials are listed in Table 1. LHG-8 (Hoya Corp, Tokyo, Japan) is a type of phosphate neodymium glass. We see that NDSG has excellent thermomechanical properties compared to the other two materials. In addition to the larger fluorescence full width at half maximum (FWHM), the thermal conductivity of NDSG is 2.2 times that of LHG-8 and the thermal shock parameter is 1.8 times that of Nd: YAG, and it has a lower nonlinear refractive index. These advantages indicate its great potential in achieving high-energy and high repetition rate lasers [4]. With the present preparation technology, the doping concentration of 
NDSG is low, which makes the emission cross section only $1.4 \mathrm{pm}^{2}$ at $1 \mu \mathrm{m}$, and the energy extraction efficiency is poor. We aim to improve this in the future, and look forward to the application of NDSG in laser amplification.

Table 1. Physical parameters of three gain materials.

\begin{tabular}{cccc}
\hline Property & NDSG & LHG-8 & Nd:YAG \\
\hline Fluorescence lifetime $(\mu \mathrm{s})$ & 376 & 315 & 230 \\
Linewidth $(\mathrm{nm})$ & 51.7 & 26.5 & - \\
Emission cross section $@ 1 \mu \mathrm{m}\left(\mathrm{pm}^{2}\right)$ & 1.4 & 4.2 & 65 \\
Thermal conductivity $\left(\mathrm{W} /(\mathrm{m} \cdot \mathrm{K})\right.$ at $\left.25^{\circ} \mathrm{C}\right)$ & 1.27 & 0.58 & 7.94 \\
Thermal expansion coefficient $\left(10^{-7} /{ }^{\circ} \mathrm{C}\right)$ & 6.5 & 115 & 82 \\
Nonlinear refractive index $\left(10^{-20} \mathrm{~m}^{2} / \mathrm{W}\right)$ & 2.89 & 3.27 & 11.79 \\
Thermal shock parameter $\left(\mathrm{W} / \mathrm{cm}^{2}\right)$ & 14.5 & 0.4 & 7.9 \\
Saturation energy density $\left(\mathrm{J} / \mathrm{cm}^{2}\right)$ & 13.47 & 4.7 & 0.66 \\
\hline
\end{tabular}

\section{Experimental Setup}

The experimental setup for the measurements presented in this paper is shown in Figure 2. The injected laser light was a pulsed circular Gaussian beam with a center wavelength of $1053 \mathrm{~nm}$ and a repetition frequency of $1 \mathrm{~Hz}$. Through the Galilean telescope with a magnification of $15 x$, the diameter of the beam was enlarged to $22.5 \mathrm{~mm}$. A 7-mm soft-edge diaphragm was used to spatially reshape the beam, changing its light-field distribution characteristics from circular Gaussian to a flat-top square beam. Hence, when the incident beam entered the pumping area of the NDSG element, the light intensity was evenly distributed. The beam energy before entering the amplifier head was measured as $2.24 \mathrm{~mJ}$.

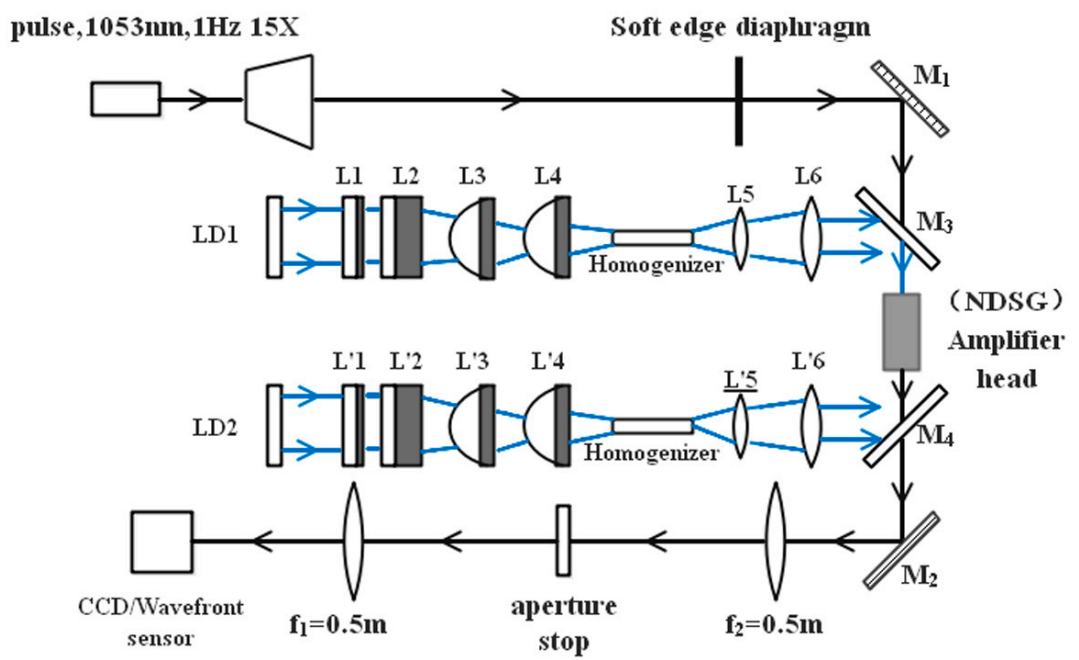

Figure 2. Layout of the LD-pumped NDSG rod laser amplification experiment. M1-M2: $45^{\circ}$ mirrors; M3-M4: dichroic mirrors; f1-f2: 4F imaging system; L1-L6, L'1-L'6: lenses.

The amplifier head, the structure of which is illustrated in Figure 3a, was made up of a NDSG rod with circulating deionized water as a cooling liquid. The NDSG rod was fixed by the O-rings at both ends of the surface, which meets the sealing conditions and achieves side cooling. As shown in Figure $3 \mathrm{~b}$, the dimensions of the NDSG cylindrical rod were $\varnothing 28 \mathrm{~mm} \times 28 \mathrm{~mm}$, and its absorption coefficient at wavelength $802 \mathrm{~nm}$ was $1.6 \mathrm{~cm}^{-1}$. The anti-reflection coatings were fabricated onto both end surfaces of the NDSG rod, which have a transmittance greater than $99.5 \%$ for the $802-\mathrm{nm}$ and 1053-nm wavelengths. The one-way transmission rate of the NDSG rod at wavelength $1053 \mathrm{~nm}$ was measured as $98.21 \%$. 


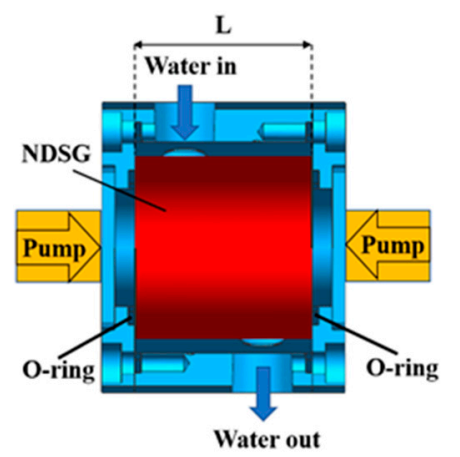

(a)

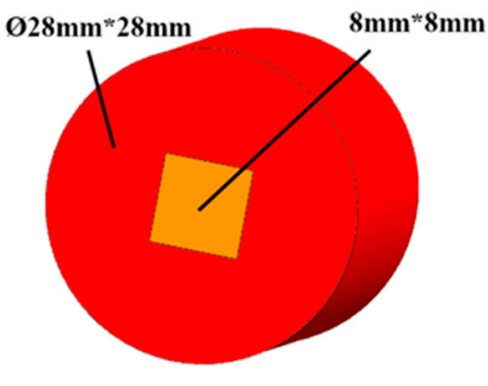

(b)

Figure 3. (a) Structure of the amplifier head, (b) NDSG rod and pump spot.

The gain module was longitudinally pumped by two symmetrical LD arrays (LD1 and LD2 in Figure 1) operating with a pulse duration of $550 \mu \mathrm{s}$. The emission area of each array was $11 \times 1.5 \mathrm{~cm}^{2}$, and the circulating deionized water was used to maintain the temperature at $25^{\circ} \mathrm{C}$. A cylindrical micro-lens was used to collimate the fast axis. For each array, the LD light was shaped into a square outline through a cylindrical lens system, and concentrated on a homogenizer of area $10 \times 10 \mathrm{~mm}^{2}$. Finally, two spherical lenses were used to image the output square pump beam, via the dichroic mirror, onto the end surface of the NDSG. The size of the pump spot was $8 \times 8 \mathrm{~mm}^{2}$, as shown in Figure $3 \mathrm{~b}$, and it was centrally positioned on the face of the NDSG rod.

A Nd:glass square rod with an absorption coefficient of $1 \mathrm{~cm}^{-1}$ was used for gain comparison. The following quantities were measured: the light energy, $E_{0}$, of the $1053 \mathrm{~nm}$ pulse signal without amplification; the laser output energy, $E_{a}$, at different pump energies; and the energy, $E_{p}$, measured through the gain medium when only the pump source was turned on. The energy of the pump light was calculated and measured using a sampling mirror and an energy meter. The gain was calculated using the formula

$$
g=\frac{\left(E_{a}-E_{p}\right)}{E_{0}}
$$

A wavefront sensor was used to measure the wavefront distortion in the NDSG rod at different pump frequencies. In order to measure the wavefront distortion of the entire pumping area, the injected light was replaced with a continuous point light source, with a center wavelength of a 1053-nm fiber laser. It was converted into parallel light by a lens with a focal length of $1.5 \mathrm{~m}$ and then transmitted through the NDSG rod. The stress-optical constant of quartz glass is $\mathrm{K}=3.5 \times 10^{-12} \mathrm{~Pa}^{-1}$ [11], indicating that the degree of deformation, and hence wavefront distortion, would be more obvious in quartz glass. We considered that the stress on the NDSG rod caused by the O-rings in Figure 3a could affect the thermally induced wavefront image. Therefore, we removed the O-rings, and used two glass plates to seal the two ends of the amplifier head, so that the circulating water could cool both end surfaces of the NDSG rod (the value of L in Figure 3a was greater than the length of the NDSG rod, and after removing the O-ring, a gap appeared and the cooling water passed through both end surfaces of the NDSG rod). Some rubber blocks were used on the side of the NDSG rod for fixing, without blocking the cooling water. This method effectively reduced the stress on the NDSG rod, compared with the method using O-rings. The wavefront distortion was then remeasured.

The uniformity of the material was recorded using a charge coupled device (CCD) imager. The $4 \mathrm{~F}$ system shown in Figure 1, consisting of two convex lenses with focal length $0.5 \mathrm{~m}$, ensured the imaging quality of the beam. In order to filter high spatial frequencies and prevent the propagation of stray light, a small-aperture diaphragm was placed in the focal plane between the two lenses. The uniformities of the NDSG and Nd:glass square rods were tested and compared. The thermal toughness was tested at the repetition frequency of $1 \mathrm{~Hz}$, and the repetition frequency was gradually increased to test the NDSG material for cracking. 


\section{Results}

The experimental values of NDSG gain are shown in Figure 4a. With a pumping energy of 7.79 J, the maximum gain is 1.16, and the corresponding absorption coefficient is $0.053 \mathrm{~cm}^{-1}$. However, for the same pumping conditions, the Nd:glass square rod achieved a gain of 2.44. The high uniformity of pump intensity distribution in the horizontal and the vertical directions is evident in Figure $4 \mathrm{~b}$.

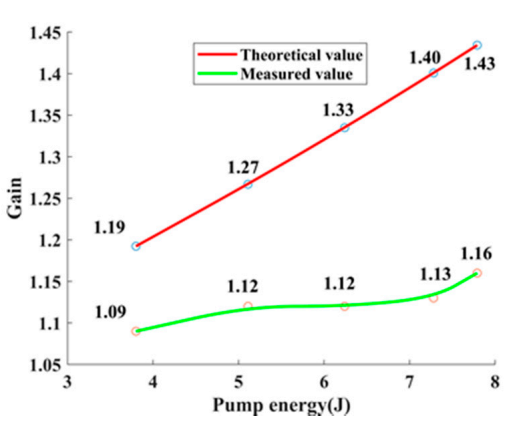

(a)

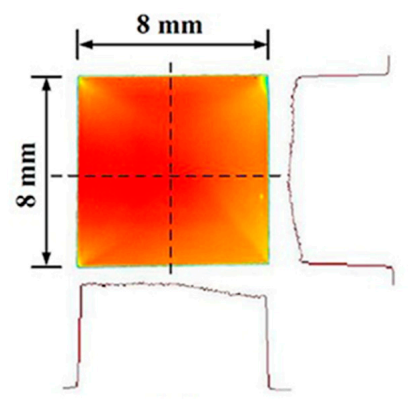

(b)

Figure 4. (a) Graph of theoretical and measured gain parameters of $\mathrm{Nd}$-doped silica glass versus pump energy and (b) pump light intensity distribution.

Because the NDSG laser is amplified in a four-level system, the ideal gain can be calculated using the equations

$$
\begin{aligned}
& g_{0}=\frac{E_{s t}}{E_{s}} \\
& E_{s}=\frac{h v}{\sigma} .
\end{aligned}
$$

Here, $g_{0}$ is the small-signal gain coefficient; $E_{s t}$ is the energy storage density; $E_{S}$ is the saturation energy density; and for the NDSG stimulated emission cross section, $\sigma=1.4 \mathrm{pm}^{2}$. The saturation energy density of NDSG, obtained by calculation, was $13.47 \mathrm{~J} / \mathrm{cm}^{2}$. The calculated energy storage density was $1.74 \mathrm{~J} / \mathrm{cm}^{2}$ (assuming a fluorescence efficiency of 53\%) when the pump energy was $7.79 \mathrm{~J}$, the absorption coefficient was $1.6 \mathrm{~cm}^{-1}$ and the pump spot size was $8 \mathrm{~mm} \times 8 \mathrm{~mm}^{2}$. Under ideal conditions, the calculated absorption coefficient is $0.32 \mathrm{~cm}^{-1}$, and, using Equation (2), the corresponding gain at $7.79 \mathrm{~J}$ is 1.43 , as seen in Figure $4 \mathrm{a}$. Comparing the theoretical and experimental results, we find that the measured gain is much lower than the calculated value, and far less than the gain of the neodymium-glass square rod. A large number of excitation particles were consumed by amplified spontaneous emission (ASE), tending to reduce the gain. However, the uniformity of the material affects the gain amplification result. Most photons fail to pass through NDSG due to scattering losses.

The wavefront distortion, when the NDSG rod was clamped using O-rings, is shown in Figure 5. The observed distribution of wavefront distortion at the pumping frequency of $1 \mathrm{~Hz}$ and a pulse energy of $7.79 \mathrm{~J}$ is characterized by central axis symmetry (Figure 5a). The peak-to-valley (P-V) wavefront distortion reached a maximum value of $6.96 \lambda(\lambda=1053 \mathrm{~nm})$ at the corners of the square. When the pump frequency was increased, the distributions of wavefront distortion were similar to those shown of Figure 5a, but the maximum values became larger, and showed a linear relationship with frequency. As shown in Figure 5b, when the frequency was increased by $1 \mathrm{~Hz}$, the increase in wavefront distortion was approximately $7.4 \lambda$, reaching $37.14 \lambda$ at $5 \mathrm{~Hz}$.

The wavefront distortion distribution, as presented in Figure 5a, was decomposed using Legendre polynomials, to yield the coefficients given in Figure 6. We found that the main distortions were due to the 1st, 4 th and 6 th terms. Of these, the 4 th and 6 th coefficients correspond to positive spherical aberration. The large stress caused by the O-rings caused a certain degree of deformation of the NDSG rod. Further, the end surfaces were not cooled; thus, heat could accumulate, causing thermal lens effects. Finally, the poor doping of NDSG would also affect the measured wavefront distortion. 


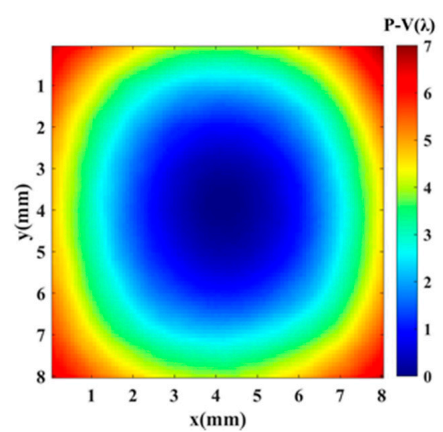

(a)

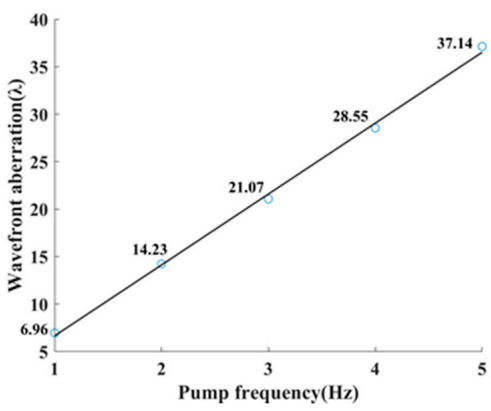

(b)

Figure 5. Peak-to-valley (P-V) wavefront distortion with fixed O-rings. (a) Distribution at pump frequency $1 \mathrm{~Hz}$. (b) Graph of maximum distortion versus pump frequency.

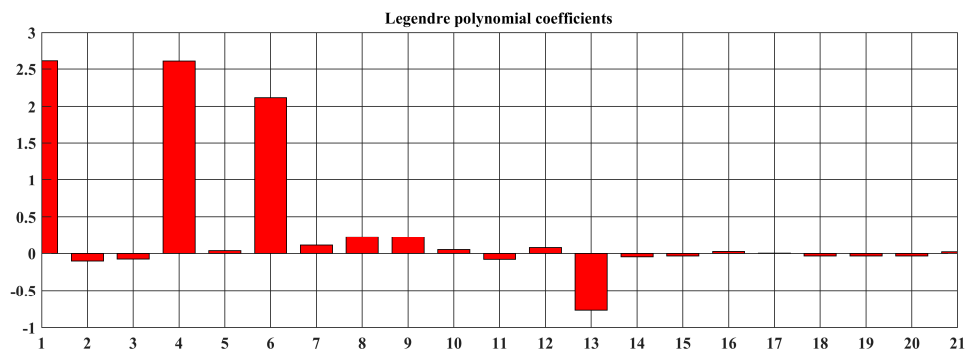

Figure 6. 2D Legendre polynomial coefficients for wavefront distortion with fixed O-rings at $1 \mathrm{~Hz}$.

In the second test, the O-rings were removed, and the resulting wavefront distortion distribution at a pump frequency of $1 \mathrm{~Hz}$ is shown in Figure 7a. Compared with Figure $5 \mathrm{a}$, the change in wavefront distortion is obvious. Here, the maximum wavefront distortion at $1 \mathrm{~Hz}$ is $2.67 \lambda$, much smaller than the $6.96 \lambda$ observed with the O-rings. A comparison of the results of these two tests reveals that stress had a great influence on the wavefront distortion for NDSG, which has a larger stress constant than that of quartz glass. The dependence of maximum wavefront distortion versus pump frequency, as shown in Figure $7 \mathrm{~b}$, displays a linear relationship similar to that in Figure $5 \mathrm{~b}$. Here, however, the values are smaller, and the maximum wavefront distortion at $5 \mathrm{~Hz}$ is $12.92 \lambda$.

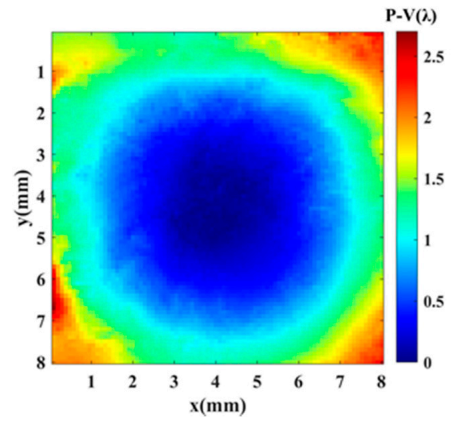

(a)

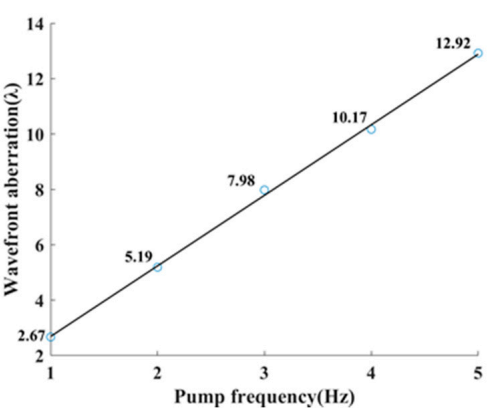

(b)

Figure 7. Peak-to-valley $(\mathrm{P}-\mathrm{V})$ wavefront distortion without fixed O-rings. (a) Distribution at pump frequency $1 \mathrm{~Hz}$. (b) Graph of maximum wavefront distortion versus pump frequency.

In Figure 8, the Legendre polynomial coefficients are given, as obtained from Figure 7a, and the distribution is very similar to that determined for the with O-rings case (Figure 6). The NDSG sealing structure was compact. Thus, even if the O-rings were removed, there was still wavefront distortion caused by partial stress at the corners. Since there was cooling water flowing over both end surfaces of the NDSG rod, the thermal lens effect was weakened, but wavefront distortion caused by fluid motion appeared. 


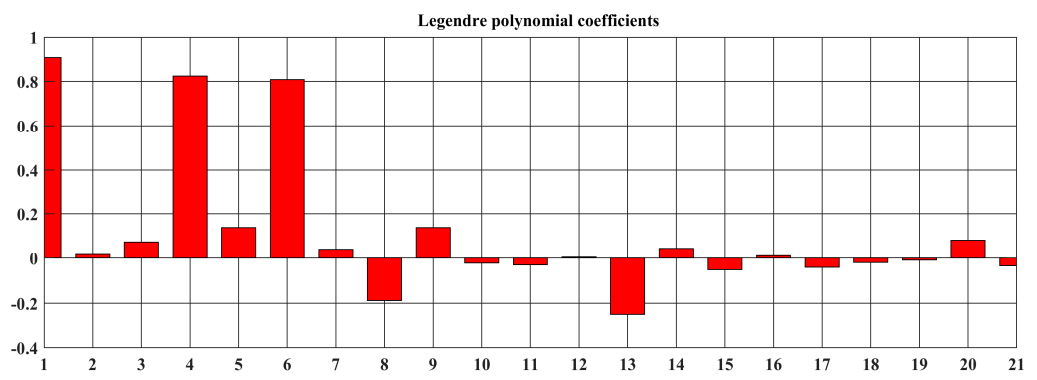

Figure 8. 2D Legendre polynomial coefficients for wavefront distortion without fixed O-rings at $1 \mathrm{~Hz}$.

The thermal stress fracture limit of a rod-shaped gain medium is given by [12]

$$
\frac{P_{a}}{L}=8 \pi R_{T}
$$

where $P_{a}$ is the limit of total heat accumulation, $L$ is the length and $R_{T}$ is the thermal shock resistance parameter. This shows that the thermal stress fracture limit is proportional to $R_{T}$. NDSG had a high thermal shock parameter of $14.5 \mathrm{~W} / \mathrm{cm}$; thus, the thermal stress fracture limit should be very high. A thermal toughness test was carried out on NDSG for comparison with the theoretical parameter. The total excitation energy of the two LD pump sources was $7.79 \mathrm{~J}$ at $1 \mathrm{~Hz}$. The pump frequency was gradually increased until it reached $25 \mathrm{~Hz}$, when the pump power was about $194 \mathrm{~W}$. At this time, the coatings on both end surfaces had melted, but the NDSG still did not crack. This showed, to a certain extent, that the thermal toughness of NDSG was very high, corresponding to its high thermal shock resistance parameter. This feature is very valuable in high-energy, high repetition rate laser amplification.

The images displayed in Figure 9 show the material uniformity, with Figure 9a showing no gain medium in the optical path, Figure $9 \mathrm{~b}$ showing the Nd:glass square rod, and Figure $9 \mathrm{c}$ showing the NDSG rod. The doping of the Nd:glass square rod was uniform, and the beam transmission uniformity was very good. However, the uneven distribution in Figure 9c shows many places where the beam failed to pass through the NDSG rod. This indicates that the Nd doping level in silica glass was not uniform, resulting in a cluster phenomenon, substantially reducing the amount of light passing through. Some parts of the beam diverged, but most were absorbed in the material, generating heat. This affected the gain and wavefront distortion.

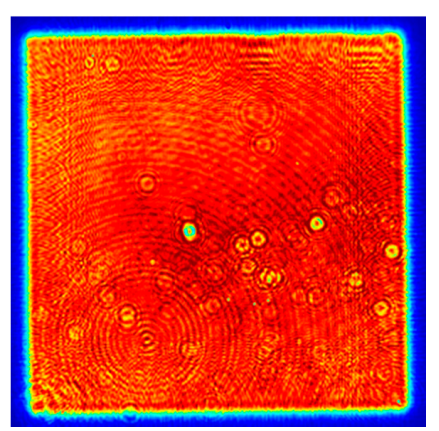

(a)

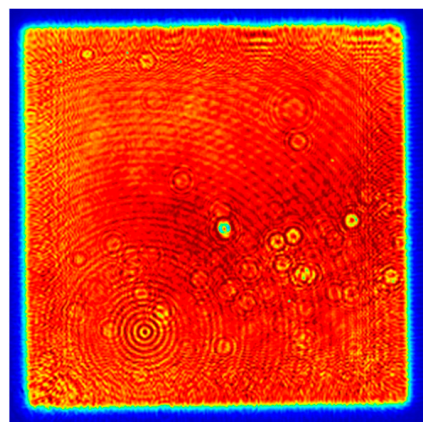

(b)

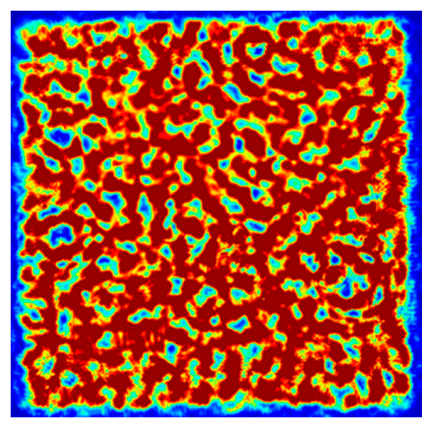

(c)

Figure 9. Material uniformity from CCD images: (a) no gain medium, (b) Nd:glass square rod and (c) NDSG rod.

\section{Conclusions}

In this study, the experimental and theoretical small-signal gain, wavefront distortion, thermal toughness and material uniformity of neodymium-doped silica glass (NDSG) as a laser medium were determined. The measured gain was 1.16 , which differed considerably from the 
calculated ideal value. The comparison with Nd:glass showed that NDSG was deficient in material uniformity. The wavefront distortion measurements demonstrated that NDSG was greatly affected by stress. At a pumping frequency of $1 \mathrm{~Hz}$, even if some stress and thermal lens effects were alleviated, the measured wavefront distortion was still relatively as large as $2.67 \lambda$. This indicates that the amplifier head structure design needs to be optimized, and the material uniformity also should be improved. These problems could be solved by improvements in the preparation technology in future research. The high thermal shock resistance parameter of NDSG $(14.5 \mathrm{~W} / \mathrm{cm})$, and other excellent optical parameters, make it a good candidate for application in high-power and high repetition frequency lasers.

Author Contributions: W.H. developed the idea for the study. S.W. provided materials. L.P. and S.J. performed the research and L.P. analyzed data and wrote the manuscript. X.L. (Xinghua Lu), J.W., W.F. and X.L. (Xuechun Li) discussed the results and revised the manuscript. All authors have read and agree to the published version of the manuscript.

Funding: This work was funded by the Chinese National Natural Science Foundation (Grant No. 61705242 and Grant No. 61975216) and Shanghai Science and Technology Foundation for Young Scholars (Grant No. 17YF1429600).

Conflicts of Interest: The authors declare no conflict of interest.

\section{References}

1. Zhang, X.; Su, L.; Cai, Q. Analysis of Thermal Effect and Experimental Test of Beam Wavefront Distortion in All Solid-State Nd:YAG Laser. Acta Opt. Sin. 2010, 30, 802-807. [CrossRef]

2. Qu, P.; Wang, S.; Guo, Z.; Cai, D.; Li, B. Analysis of Interaction Between Thermal Effect and Pump Optical Field Distribution in High Power Solid-State Laser. Acta Opt. Sin. 2014, 34, 1114002. [CrossRef]

3. Sato, T.; Fujimoto, Y.; Ueda, T.; Fujinoki, A.; Okada, H.; Yoshida, H.; Sumimura, K.; Nakatsuka, M. Laser Oscillation of Nd-Doped Silica Glass with High Thermal Shock Parameter. Jpn. J. Appl. Phys. 2006, 45, 6936-6939. [CrossRef]

4. Stokowski, S.E.; Saroyan, R.A.; Weber, M.J. Nd-Doped Laser Glass Spectroscopic and Physical Properties; Technical Report UCRL-TR-208148; University of California Radiation Laboratory: Berkeley, CA, USA, 2004. [CrossRef]

5. Fujimoto, Y.; Yoshida, H.; Nakatsuka, M.; Ueda, T.; Fujinoki, A. Development of Nd-doped Optical Gain Material Based on Silica Glass with High Thermal Shock Parameter for High-Average-Power Laser. Jpn. J. Appl. Phys. 2005, 44, 1764-1770. [CrossRef]

6. Sato, T.; Fujimoto, Y.; Okada, H.; Yoshida, H.; Nakatsuka, M.; Ueda, T.; Fujinoki, A. 40 J class laser oscillation of Nd-doped silica glass with high thermal shock parameter. Appl. Phys. Lett. 2007, 90, 221108. [CrossRef]

7. Galant, E.I.; Kondrat'ev, Y.N.; Przhevuskii, A.K.; Prokhorova, T.I.; Tolstoi, M.N.; Shapovalov, V.N. Stimulated Emission of Neodymium Ions in Quartz Glass. J. Exp. Theor. Phys. Lett. 1973, 18, 372.

8. Arai, K.; Namikawa, H.; Kumata, K.; Honda, T.; Ishii, Y.; Handa, T. Aluminum or phosphorus co-doping effects on the fluorescence and structural properties of neodymium-doped silica glass. J. Appl. Phys. 1986, 59, 3430-3436. [CrossRef]

9. Thomas, I.M.; Payne, S.A.; Wilke, G.D. Optical properties and laser demonstrations of Nd-doped sol-gel silica glasses. J. Non-Cryst. Solids 1992, 151, 183-194. [CrossRef]

10. Fujimoto, Y.; Nakatsuka, M. A novel method for uniform dispersion of the rare earth ions in $\mathrm{SiO}_{2}$ glass using zeolite X. J. Non-Cryst. Solids 1997, 215, 182-191. [CrossRef]

11. Davis, M.J.; Hayden, J.S. Stress-optic measurements of SCHOTT laser glass. In Proceedings of the Solid State Lasers XXVIII Technology Devices, San Francisco, CA, USA, 5-7 February 2019; Volume 10896, p. 108961E. [CrossRef]

12. Koechner, W. Solid-State Laser Engineering; Springer Science and Business Media LLC: Berlin, Germany, 1999; Volume 1, p. 412.

(C) 2020 by the authors. Licensee MDPI, Basel, Switzerland. This article is an open access article distributed under the terms and conditions of the Creative Commons Attribution (CC BY) license (http://creativecommons.org/licenses/by/4.0/). 